\title{
The Möbius algebra as a Burnside ring
}

\author{
Dedicated to Professor Hiroshi Nagao \\ on his sixtieth birthday \\ By Tomoyuki YosHidA \\ (Received May 10, 1984)
}

\section{Introduction}

The Burnside ring $\Omega(G)$ of a finite group $G$ is the Grothendieck ring of the category of finite $G$-sets with respect to disjoint unions and cartesian products. It was first introduced by Solomon [1967] and has been ever since studied by many people in several fields. Especially Dress [1971], [1973] show that the Grothendieck rings of "based categories" possesses similar properties to the Burnside rings of finite groups (the existence of an embedding mapping $\phi$, Mackey property, Frobenius reciprocity, Dress induction theorem, etc.) and he applied them to abstract induction theory. However there is room for development in his theory. First an $\mathscr{S}_{f}$-topos (that is, a topos with finite hom-sets) is much better than a based category as a base of Dress theory. (On topos theory, refer to Johnstone [1977].) Secondly many properties of the Burnside rings are brought not from the distributive property of finite $G$-sets but from a few properties of the category of finite $G$-sets (i. e. the regularity of the category, particularly unique epimono factorization, c.f. [Gri. 1971]), and so it is possible to define and study the Burnside ring even for a regular category.

In view of topos theory, combinatorial theory is theory of $\mathscr{S}_{f}$-toposes which satisfy Axiom of Choice, and finite group theory is theory of Boolean $\mathscr{S}_{f}$-toposes. When we study the Burnside rings of toposes and regular categories, we are interested in two extremal cases, that is, finite semigroups and finite posets (and the functor categories of them to the category of finite sets). In this paper, we try the case of finite posets. (I am preparing papers about the general case and the semi-group case).

In Section 2, we introduce the concept of $P$-sets (a $P$-set is a finite-setvalued contravariant functor on the poset $P$ viewed as a category) and give three definitions of the Burnside rings of a poset, that is, the $K_{0}$-ring, the $G_{0}$-ring and $Z[P]$. In Theorem 2, we show that for a finite poset $P, G_{0}(P)$ and $Z[P]$ are both isomorphic to the Möbius ring $A(P)$. In Section 3, we 
study some adjoint functors between the category of $P$-sets and mappings between Möbius rings induced from them. Following Baclawski [1979], we can regard a $P$-set as a sheaf of finite sets on $P$, where $P$ is a topological space in which ideals are open sets. Thus there corresponds the $P$-set $X$ to a "locally homeomorphism" $X \rightarrow P$ (cf. [T. 1975]). Introducing the concept of locally homeomorphism of posets, we can show that the Möbius ring functor makes a Green functor, that is, a multiplicative Mackey functor satisfying the Frobenius reciprocity (Theorem 4). (On Mackey functors, refer to Dress [1973].) Then we interest in transfer theorems and induction theorems for Möbius rings. We prove Dress induction theorem and a kind of transfer theorem in Theorem 5 and 6. Finally we mention span rings of posets.

Notation. We employ the usual terminology for posets and categories as in [A. 1979], [M. 1971]. We denote the categories of finite sets, abelian groups, posets by $\mathscr{S}_{f}, \mathscr{A} b, \mathscr{O}$, respectively. For a category $\mathscr{C}$ and an object $X$, the comma category $\mathscr{C} / X$ is the category with objects all morphisms to $X$, and with morphisms $f$ of $(a: A \rightarrow X)$ to $(b: B \rightarrow X)$ those morphisms $f$ of $A$ to $B$ in for which $b f=a$. We regard a poset as a category as usual ([M. 1971, p. 11]). Refer to [A. 1979] for the $\zeta, \mu, \delta$ functions on posets. The disjoint union (coproduct) and direct product of posets $P$ and $Q$ are denoted by $P+Q$ and $P \times Q$.

\section{The Burnside rings of finite posets}

After this let $P, Q$ be finite posets (= partially ordered sets). The Möbius ring $A(P)$ of $P$ is a free abelian group on $P$ with product defined on basis by

$$
x \cdot y=\sum_{z}\left(\sum_{t \leqq x, y} \mu(z, t)\right) z,
$$

where $\mu$ is the Möbius function on $P$.

We regard the poset $P$ as a category as in [M. 1971, p. 11]. By $\hat{P}$ we denote the category of contravariant functors of $P$ to $S_{f}$, the category of finite sets. An object of $\hat{P}$ is called a $P$-set, and a morphism is called a $P$-map. Thus a $P$-set $X$ consists of $P$-indexed finite sets $X^{i}$ and restriction maps

$$
\operatorname{res}_{i}^{j}: X^{j} \longrightarrow X^{i}: x \longrightarrow x \mid i, \quad i \leqq j
$$

satisfying the conditions :

(i) $x \mid i=x$ for $i \in P, x \in X^{i}$; 
(ii) $(z \mid j)|i=z| i$ for $i \leqq j \leqq k, z \in X^{k}$.

Furthermore, a $P$-map $f: X \rightarrow Y$ consists of a family $\left(f^{i}: X^{i} \rightarrow Y^{i}\right)_{i \in P}$ which is compatible with restriction maps. The category $\hat{P}$ is an $\mathscr{S}_{f}$-topos (Johnstone [1977]) and it is finitely complete and finitely cocomplete ((co-) limits are constructed elementwise).

Definition of $K_{0}$ and $G_{0}$. Let $\mathcal{E}$ be a skeletally small category with finite coproducts. Then $K_{0}(\mathcal{E})$ is the free abelian group on isomorphism classes $[X]$ modulo the relations $[X+Y]=[X]+[Y]$. Let $M(\varepsilon)$ be the subgroup of $K_{0}(\mathcal{E})$ generated by elements of the form $[A]-[B]-[C]+[D]$ for some pushout diagram

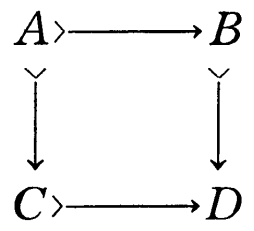

with four monomorphisms; and then we set $G_{0}(\varepsilon)=K_{0}(\varepsilon) / M(\varepsilon)$.

For example, if $\varepsilon$ is the category of finitely generated $R$-modules for a ring $R$, then $G_{0}(\varepsilon)$ coincides with the ordinary Grothendieck group $G_{0}(R)$; moreover if $\varepsilon$ is the category of finite $G$-sets for a finite group $G$, then $K_{0}(\varepsilon)$ as well as $G_{0}(\varepsilon)$ is the Burnside ring of $G$.

In general if $\mathcal{E}$ is a small topos (e.g. $\hat{P}$ ), then $K_{0}(\mathcal{E})$ has the structure of a commutative ring $([X] \cdot[Y]=[X \times Y])$ and $M(\mathcal{E})$ is an ideal of $K_{0}(\varepsilon)$, and so $G_{0}(\varepsilon)$ is also a commutative ring.

Definition of the Burnside ring $Z[\mathscr{A}]$. Let $\mathscr{A}$ be a finite skeletal category. Let $Z[\mathscr{A}]$ be the free abelian group with basis $\operatorname{Obj}(\mathscr{A})$, and let $Z^{\mathscr{A}}$ be the $\operatorname{Obj}(\mathscr{A})$-indexed direct product. Then we have a linear map

$$
\phi: Z[\mathscr{A}] \longrightarrow Z^{\mathscr{A}}: A \longmapsto(|\operatorname{hom}(I, A)|)_{I \in \mathscr{A}} \text {. }
$$

Assume that the following conditions hold:

(a) $\phi$ is injective.

(b) The image of $\phi$ is a subring of $Z$.

Under this assumption, $\boldsymbol{Z}[\mathscr{A}]$ becomes a commutative ring with an injective ring homomorphism $\phi$. This ring $Z[\mathscr{A}]$ is also called the Burnside ring of $\mathscr{A}$.

If $\mathscr{A}$ is a skeletal finite full subcategory of a regular category (e. g. toposes, abelian categories, posets) and it is closed with respect to epimorphic images, then (a) and (b) hold. In case where $\mathscr{A}$ is a skeleton of the category of transitive $G$-set, then the Burnside ring $Z[\mathscr{A}]$ defined as above is isomorphic to the ordinary Burnside ring of $G$. 
Let $P$ be a finite poset. We set

$$
K_{0}(P)=K_{0}(\hat{P}), \quad G_{0}(P)=G_{0}(\hat{P}) .
$$

All of rings $K_{0}(P), G_{0}(P), Z[P]$ are suitable to be the "Burnside rings" of $P$. For example the embedding theorem for $K_{0}$ (cf. [D. 1973, Proposition 4. 3]) has the following form :

THEOREM 1. The ring homomorphism

$$
\phi=\left(\phi^{C}\right): K_{0}(P) \longrightarrow Z^{\operatorname{con}(\hat{P})}:[X] \longrightarrow\left(\left|\operatorname{hom}_{P}(C, X)\right|\right)_{C}
$$

is an injection, where $\operatorname{Con}(\hat{P})$ is the representatives of isomorphism classes of connected P-sets. (A P-set is called connected provided it is not the coproduct of any two nonempty $P$-subsets.)

Similarly as in Yoshida [198?], this theorem follows easily from an LDUdecomposition of the Hom-set matrix $(|\operatorname{hom}(A, B)|)$; so we omit the proof. ${ }^{\text {) }}$ However, $K_{0}(P)$ is in general too large; in fact if $P$ is not discrete, then $K_{0}(P)$ contains a polynomial ring with countably many variables. So we are mainly interested in $G_{0}(P)$ and $Z[P]$; the embedding theorems for them are simple.

THEOREM 2. Let $P$ be a finite poset viewed as a category. Then the following hold:

(i) The Burnside ring $Z[P]$ can be defined and it has the same multiplication table as the Möbius ring $A(P)$.

(ii) There are ring isomorphism $\phi, \phi^{\prime}, \bar{\eta}$ with $\phi=\phi^{\prime} \bar{\eta}$ as follows :

$$
\begin{aligned}
& \phi: A(P) \longrightarrow Z^{P}: i \longmapsto(\zeta(j, i))_{j \in P}, \\
& \phi^{\prime}: G_{0}(P) \longrightarrow Z^{P}:[X] \longrightarrow X \longrightarrow X_{j \in P}, \\
& \bar{\eta}: A(P) \longrightarrow\left[G_{0}(P): i \longmapsto\right.
\end{aligned}
$$

Proof. (i) In the category $P$,

$$
\left|\operatorname{hom}_{P}(i, j)\right|=\zeta(i, j)= \begin{cases}1 & \text { if } i \leqq j \\ 0 & \text { otherwise }\end{cases}
$$

Since the matrix $(\zeta(i, j))_{i, j \in P}$ has clearly an inverse in $G L(|P|, Z)$, the linear map

$$
\phi: Z[P] \longrightarrow Z^{P}: j \longrightarrow(\zeta(i, j))_{i}
$$

1) Add in proof. The similar result is proved by Lovász (Acta Sci. Math. (Szeged), 33 (1972), 319-322). 
is an isomorphism of abelian groups. Thus the Burnside ring $\boldsymbol{Z}[P]$ can be defined as a ring isomorphism to $Z^{P}$ through $\phi$, and so the multiplication $i \cdot j$ is defined by $\phi^{-1}(\phi(i) \cdot \phi(j))$. Assume that $i \cdot j=\Sigma_{k} a(k) k$, so that acting $\phi$, we have that

$$
\Sigma_{k} a(k) \zeta(l, k)=\zeta(l, i) \zeta(l, j) \quad \text { for } \quad l \in P .
$$

By the Möbius inversion,

$$
\begin{aligned}
a(m) & =\Sigma_{k, l} a(k) \mu(m, l) \zeta(l, k) \\
& =\Sigma_{l} \mu(m, l) \zeta(l, i) \zeta(l, j) .
\end{aligned}
$$

Hence we have

$$
i \cdot j=\sum_{k}\left(\sum_{l \leqq i, j} \mu(k, l)\right) k
$$

This multiplication coincides to one of the Möbius ring $A(P)$.

(ii) Since the functor $X \mapsto X^{j}$ preserves pushout diagrams and monomorphisms, it induces a ring homomorphism

$$
\phi^{\prime}: G_{0}(P) \longrightarrow Z^{P}:[X] \longrightarrow\left(\left|X^{j}\right|\right)_{j \in P},
$$

Furthermore, the Yoneda functor $P \rightarrow \hat{P}: i \mapsto h_{i}$ induces a linear map

$$
\eta: A(P) \longrightarrow K_{0}(P): i \longmapsto\left[h_{i}\right] \text {. }
$$

Since $\phi^{\prime}\left(\left[\mathrm{h}_{i}\right]\right)=(\zeta(j, i))_{j \in P}=\phi(i)$, we have the commutative diagram :

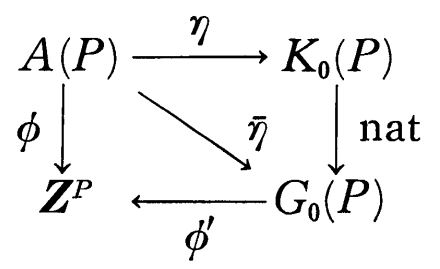

A nonempty $P$-set $A$ is called irreducible provided $A$ has a unique maximal $P$-subset. We shall first prove that any $[X] \in G_{0}(P)$ is a linear combination of some classes $[A]$ of irreducible $P$-sets $A$ in $G_{0}(P)$. This follows from an easy induction argument on $\Sigma_{i}\left|X^{i}\right|$. In fact if $X$ has two maximal $P$-subsets $Y$ and $Z$, and $X=Y \cup Z$, and so

$$
[X]=[Y \cup Z]=[Y]+[Z]-[Y \cap Z] \text { in } G_{0}(P) .
$$

Since by induction hypothesis $[Y]$ and $[Z]$ are already linear combinations of classes of irreducible $P$-sets, so is $[X]$, as required. Now irreducible $P$ sets are representable, that is, if $A$ is irreducible then there exists $i$ in $P$ such that $A$ is isomorphic to $h_{i}$; in fact, $h_{i}$ 's are (pro-) generators in $P$ and each $h_{i}$ has no nontrivial quotient objects. Thus we have that $G_{0}(P)$ is 
generated by $\left[h_{i}\right], i \in P$. This means that $\bar{\eta}: i \rightarrow\left[h_{i}\right]$ is surjective. Since $\phi=\phi^{\prime} \bar{\eta}$ is a ring isomorphism and $\phi^{\prime}$ is a ring homomorphism, we conclude from the surjective of $\bar{\eta}$ that $\phi^{\prime}$ and $\bar{\eta}$ are ring-isomorphisms. The theorem is proved.

By the Möbius inversion, we have that $\phi^{\prime}\left(e_{i}\right)=\left(\delta_{i, j}\right)_{j \in P}$. Thus by the theorem, primitive idempotents of $G_{0}(P)$ have the form

$$
e_{i}=\Sigma_{j} \mu(j, i)\left[h_{j}\right]
$$

It is well-known that primitive idempotents of the Möbius ring $A(P)$ has the form

$$
\delta_{i}=\Sigma_{j} \mu(j, i) j
$$

and that $j=\Sigma_{i \leqq j} \delta_{i}$.

Let $k$ be a principal ideal domain (e.g., a field). We write $k \hat{P}$ for the functor category of $P^{o p}$ to the category of finitely generated $k$-modules. The category $k \hat{P}$ is abelian and tensor products are defined; thus we obtain the Grothendieck ring $G_{0}(k \hat{P})$. The free functor

$$
F: \hat{P} \longrightarrow k \hat{P}: X \longmapsto \mid k X\left(=\left(k X^{i}\right)_{i \in P}\right)
$$

preserves finite colimits and monomorphisms; and it maps products to tensor products; and so it induces a ring homomorphism

$$
\pi: G_{0}(P) \longrightarrow G_{0}(k \hat{P}):[X] \longmapsto[k X] .
$$

Furthermore there is also a ring homomorphism

$$
\chi: G_{0}(k \hat{P}) \longrightarrow Z^{P}:[M] \longmapsto\left(\operatorname{rank}_{k} M^{i}\right)_{i \in P} .
$$

By Theorem 2 (ii), $\phi^{\prime}=\chi \pi$ is an isomorphism; and by Baclawski [1979, Lemma 5], $\pi$ is surjective. Thus the above $\pi$ and $\chi$ are isomorphisms, and so

$$
G_{0}(k \hat{P}) \cong A(P) \cong G_{0}(P) \cong Z^{P} .
$$

\section{Functorial properties of Möbius rings}

In this section, we study some maps between Möbius rings induced from an order preserving map. We will show that the Möbius rings give a contravariant functor $A^{*}$ of $\mathcal{O}$, the category of finite posets and order-preserving maps, to rings and a functor $A_{1}$ of $\mathscr{O}_{l h}$, the category of finite posets and locally homeomorphisms, to abelian groups and furthermore that the pair of these functors satisfies Mackey property and Frobenius reciprocity (Theorem 4). 
Through this section, let $f: P \rightarrow Q$ be an order preserving map between posets $P, Q$. For any $q \in Q$, we define subposets of $P$ as follows :

$$
\begin{aligned}
f / q: & =\{p \in P \mid f(p) \leqq q\}, \\
q \backslash f: & =\{p \in P \mid q \leqq f(p)\} .
\end{aligned}
$$

Adjoint functors $f_{1} \dashv f^{*} \dashv f_{*}$. The map $f: P \rightarrow Q$ induces an inverse image functor which is defined by:

$$
f^{*}: \hat{Q} \longrightarrow \hat{P}:\left(B^{j}\right)_{j \in Q} \longrightarrow\left(B^{f(i)}\right)_{i \in P}
$$

This functor has left and right adjoint functors (MacLane [1971, Chapter X]) :

$$
\hat{P} \underset{f_{*}}{\stackrel{f_{1}}{\rightleftarrows}} \hat{Q}, \quad f_{!} \dashv f^{*} \dashv f_{*}
$$

The right adjoint $f_{*}$ is called a direct image functor. These three functors are constructed on objects as follows :

(i) $f_{1}(X)^{a}=\prod_{i \in a \backslash f}\{i\} \times X^{i} / \sim$,

where $\sim$ is the least equivalence relation such that $(i, x) \sim(j, y)$ whenever $i \leqq j$ and $y \mid i=x$.

(ii) $f^{*}(Y)^{i}=Y^{f(i)}$.

(iii) $f_{*}(X)^{a}=\left\{\left(x_{i}\right) \in \prod_{i \in f / a} X^{i}\left|x_{j}\right| i=x_{i}\right.$ for $\left.i \leqq j\right\}$.

The inverse image functor $f^{*}$ preserves limits and colimits, and so $f^{*}$ induces ring homomorphisms $f^{*}: K_{0}(Q) \rightarrow K_{0}(P)$ and $f^{*}: A(Q) \rightarrow A(P)$ by Theorem 2. Let $a \in Q$ and let $\delta_{a}\left(=\Sigma_{b} \mu(b, a) b\right)$ be the primitive idempotent of $A(Q)$. Then in $A(P)$, we have

$$
\begin{aligned}
& f^{*}(a)=\sum_{i \in P}\left(\sum_{j \in f / a} \mu(i, j)\right) i, \\
& f^{*}\left(\delta_{a}\right)=\sum_{i \in f^{-1}(a)} \boldsymbol{\delta}_{i} .
\end{aligned}
$$

In fact it follows from Möbius inversion that the isomorphism $\phi: j \mapsto(\zeta(i, j))_{i}$ (Theorem 2 (ii)) maps both sides to the same elements.

Galois maps. The direct image functor $f^{*}$ does not always preserve coproducts. (It preserves coproducts if and only if $q \backslash f$ is connected for any $q \in Q$.) Greene [1973] gives a condition that the direct image functor $f^{*}$ induces a ring homomorphism between Möbius rings. Let $f: P \rightarrow Q$ be an 
Galois map (that is, $f$ has a right adjoint $g: Q \rightarrow P$ ). Then $f^{*}$ has a right adjoint $g^{*}$, and so $f^{*} \cong g^{*}$ preserves not only limits but also colimits, whence $f_{*}$ induces ring homomorphisms $f_{*}: K_{0}(P) \rightarrow K_{0}(Q)$ and $f^{*}: A(P) \rightarrow A(Q)$. The last ring homomorphism is the same as in Greene [1973, Theorem 2]. In fact, for any $i \in P$, we have

$$
\begin{array}{rlrl}
f_{*}(i) & =g^{*}(i)=\sum_{a}\left(\sum_{b \in g / i} \mu(a, b)\right) a & \\
& =\sum_{a}\left(\sum_{b \leq f(i)} \mu(a, b)\right) a & & \text { by adjunction } \\
& =\sum_{a} \delta(a, f(i)) a & & \text { by Möbius inversion } \\
& =f(i) . & &
\end{array}
$$

Adjount functors $\Sigma_{f} \dashv f^{*} \dashv \Pi_{f}$. Let $f: X \rightarrow Y$ be a $P$-map between $P$. sets. Then by the fundamental theorem of topos theory (Johnstone [1977, Theorem 1.42 and Corollary 1.44]), $f$ induces three functors as follows:

$$
\hat{P} / X \underset{\Pi_{f}}{\stackrel{\Sigma_{f}^{*}}{\rightleftarrows}} \hat{P} / Y, \quad \Sigma_{f} \dashv f^{*} \dashv \Pi_{f} .
$$

The pullback functor $f^{*}$ is defined as the operatioon of pulling back along $f$. The functor $\Sigma_{f}$ is defined by the composition $a \mapsto f a$.

An order preserving map $h: P \rightarrow Q$ is called a locally homeomorphism if $h$ gives a bijection of $J_{x}(=\{y \in P \mid y \leqq x\})$ to $J_{h(x)}$ for each $x \in P$. For example, an order preserving injection $h$ of $P$ to $Q$ is locally homeomorphism if and only if $P$ is isomorphic to an ideal of $Q$ through $h$. Let $\mathcal{O}_{l n}$ be the category of finite posets and locally homeomorphisms. For a $P$-set $X$, the set

$$
\tilde{X}=\coprod_{i \in P}\{i\} \times X^{i}
$$

becomes a poset by

$$
(i, x) \leqq(j, y) \quad \text { iff } \quad i \leqq j \text { and } y \mid i=x .
$$

Then the projection $p_{X}: \tilde{X} \rightarrow P:(i, x) \mapsto i$ is a locally homeomorphism; and each $P$-map $f: X \rightarrow Y$ induces a locally homeomorphism

$$
\tilde{f}: \tilde{X} \longrightarrow \tilde{Y}:(i, x) \longrightarrow\left(i, f^{i}(x)\right)
$$

Conversely let $A \stackrel{h}{\rightarrow} P$ be a locally homeomorphism; then for any $i \leqq j$ in $P$ and $a \in h^{-1}(j)$, the set $h^{-1}(i) \cap J_{a}$ consists of a unique element $a \mid i$; and 
so we obtain a $P$-set $\left(h^{-1}(i)\right)_{i \in P}$ of which restriction map is defined by $a \mapsto a \mid i$. These correspondence gives an equivalence

$$
\Theta_{l h} / P \cong \hat{P} \text {. }
$$

Furthermore, for each $P$-set $X$, this equivalence gives

$$
\hat{P} / X \cong \hat{X} \quad\left(\cong \mathcal{O}_{l n} / \tilde{X}\right) .
$$

These equivalences are natural with respect to pullback functors and inverse image functors. Thus two functors $P \mapsto \hat{P}$ and $P \mapsto \mathbb{O}_{l n} / P$ from posets to the category of toposes and essential geometric morphisms are equivalent. Since $\Sigma_{f}: \hat{P} / X \rightarrow \hat{P} / Y$ induced by a $P$-map $f$ preserves not only colimits but also pullbacks (and so monomorphisms), it follows from Theorem 2 that any locally homeomorphism $h: P \rightarrow Q$ induces an additive homomorphism

$$
h_{1}: A(P) \longrightarrow A(Q): x \longmapsto \longrightarrow h(x) \text {. }
$$

Hence the Möbius ring functor $A$ is a functor which to each locally homeomorphism $h$ assigns a ring homomorphism $h^{*}$ and an additive homomorphism $h_{1}$ with $h^{*}$ contravariant and $h_{1}$ covariant.

LеммA 3. In a pullback diagram of finite posets

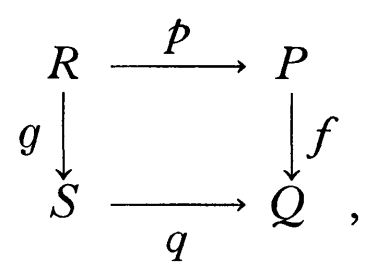

if $q$ is a locally homeomorphism, then so is $p$ and the following diagram is commutative without natural isomorphism:

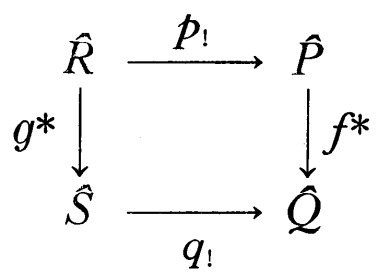

Proof. There corresponds a $Q$-set $Y$ to a locally homeomorphism $q: S \rightarrow Q$, and so we may assume that $q=p_{Y}: S=\tilde{Y} \rightarrow Q$. Set $X=f *(Y) \in \hat{P}$. Then we have a pullback diagram

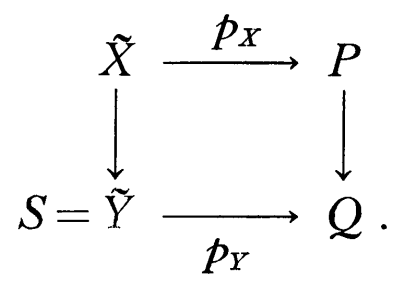


Thus it follows from the uniqueness of pullbacks that $p$ is a locally homeomorphism. We may beforehand assume that $R=\tilde{X}, p=p_{X}, q=q_{Y}$. Consider the following diagram :

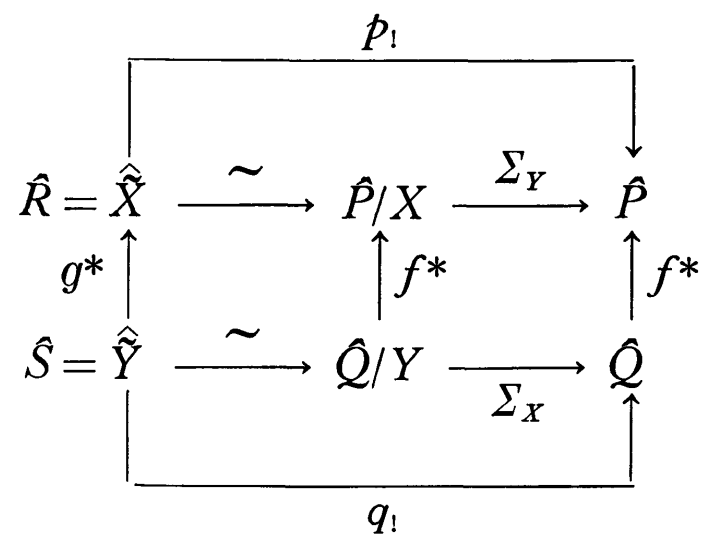

Two inner squares and two triangles are commutative, and so the outer square is also commutative, proving the lemma.

Theorem 4. (i) The pair $A=\left(A^{*}, A_{1}\right)$ of the functors

$$
\begin{aligned}
& A^{*}: \mathscr{O}_{l h}{ }^{o p} \longrightarrow \mathscr{A} b: P \longmapsto \longrightarrow A(P), f \longmapsto \longrightarrow f^{*}, \\
& A_{1}: \mathscr{O}_{l h} \longrightarrow \mathscr{A} b: P \longmapsto A(P), f_{1} \longrightarrow f_{1}
\end{aligned}
$$

is a Green functor (Dress [1971], [1973]), that is, the following hold:

(a) (Mackey decomposition) For any pullback diagram

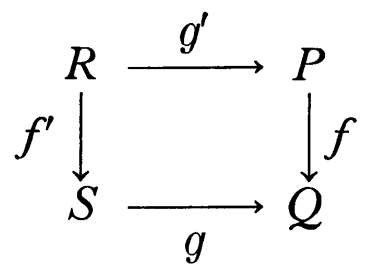

in $\mathfrak{O}_{l h}$, the following diagram is commutative:

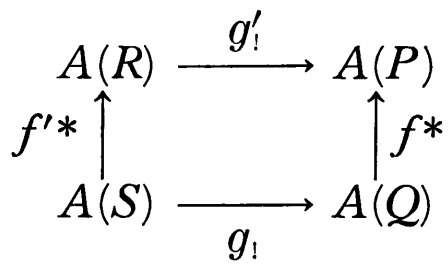

(b) If $\stackrel{i}{\rightarrow} P+Q \stackrel{j}{\leftarrow} Q$ is a coproduct diagram in $\mathscr{O}_{l h}$ then

$$
A(P) \stackrel{i^{*}}{\underset{i_{1}}{\leftrightarrows}} A(P+Q) \underset{j_{!}}{\stackrel{j^{*}}{\rightleftarrows}} A(Q)
$$

is a biproduct diagram of abelian groups. 
(c) For any locally homeomorphism $f$, the map $f^{*}$ is a ring homomorphism.

(d) (Frobenius reciprocity) Let $f: P \rightarrow Q$ be a locally homeomorphism. Then for any $x \in A(P)$ and $y \in A(Q)$,

$$
f_{1}(x) y=f_{1}\left(x f^{*}(y)\right) \text {. }
$$

(ii) If a poset $P$ is the union of two ideals $I$ and $J$ of $P$ with injections $i: I \rightarrow P$ and $j: J \rightarrow P$, then

$$
\left(\mathrm{id}_{A(P)}-i_{1} i^{*}\right)\left(\mathrm{id}_{A(P)}-j_{1} j^{*}\right)=0 \text { on } A(P) .
$$

Proof. (a) This is a special case of Lemma 3. (b) Set $R=P+Q$. Then there exist $R$-sets $X$ and $Y$ which correspond to locally homeomorphism $i: P \rightarrow R$ and $j: Q \rightarrow R$, that is, $\tilde{X}=P, \tilde{Y}=Q, i=p_{X}, j=p_{Y}$. Thus we have a coproduct diagram $X \rightarrow \mathbf{1} \leftarrow Y$, where 1 is a final object in $\hat{R}$. Now consider the functors

$$
\hat{R} / X \underset{\Sigma_{X}}{\stackrel{X^{*}}{\leftrightarrows}} \hat{R} \underset{\Sigma_{Y}}{\stackrel{Y^{*}}{\rightleftarrows}} \hat{R} / Y .
$$

Then in functor categories, we have, without natural isomorphisms,

$$
\begin{array}{rlrl}
1_{\hat{R}} & =\Sigma_{X} X^{*}+\Sigma_{Y} Y^{*}, \\
X^{*} \Sigma_{X} & =1_{\hat{R} / X}, & Y^{*} \Sigma_{Y} & =1_{\hat{\hat{R}} / Y}, \\
Y^{*} \Sigma_{X} & =0, \quad X^{*} \Sigma_{Y}=0 .
\end{array}
$$

Applying $G_{0}$ on these equalities, we see that the two lines of the following diagram are biproduct diagrams :

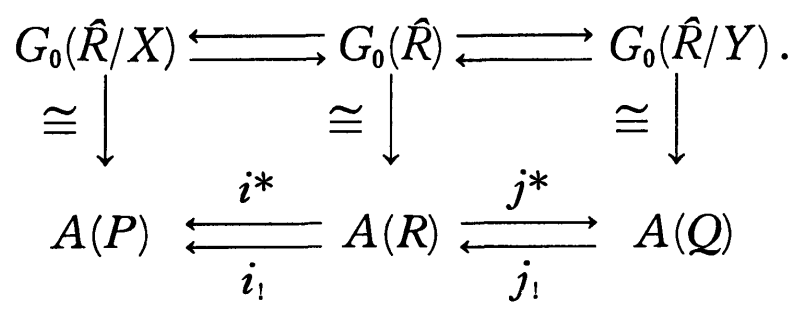

(c) This has been already shown.

(d) Let $A$ be a $Q$-set which corresponds to the locally homeomorphism $f$ : $P \rightarrow Q$, so that $A(P) \cong G_{0}(\hat{Q} / A)$ and $A(Q) \cong G_{0}(\hat{Q})$. Thus it will suffice to show that Frobenius reciprocity for $G_{0}(\hat{Q} / A) \rightleftarrows G_{0}(\hat{Q})$ holds. Let $X \stackrel{x}{\rightarrow} A$ be an object of $\hat{Q} / A$ and let $Y$ be an object of $\hat{Q}$. Then we have

$$
\Sigma_{\Delta}(X \stackrel{x}{\longrightarrow} A) \times Y=X \times Y
$$


Furthermore since

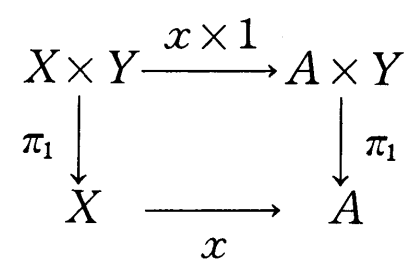

is a pullback diagram,

$$
\Sigma_{A}\left((X \stackrel{x}{\longrightarrow} A) \times\left(A \times Y \stackrel{\pi_{1}}{\longrightarrow} A\right)\right)=X \times Y .
$$

Hence we have

$$
\Sigma_{A}(x) \times Y=\Sigma_{A}\left(x \times f^{*}(Y)\right) .
$$

This shows the Frobenius reciprocities for $K_{0}, G_{0}$ and for the Möbius rings $A$.

(ii) This follows easily from the direct verification.

REMARK. Similarly, the $K_{0}$-functor becomes a Green functor.

Similarly as in the case of the Burnside ring of a finite group, there is an induction theorem and a transfer theorem (or fusion theorem) for Möbius rings. First Dress induction theorem (Dress [1971, Theorem 7. 1], Yoshida [1983]) which generalizes Artin induction theorem holds in the best form.

Theorem 5. (Dress induction theorem) Let $h: P \rightarrow Q$ be a locally homeomorphism and set

$$
\begin{aligned}
I_{P}(Q): & =\operatorname{Im}\left(h_{!}: A(P) \longrightarrow A(Q)\right), \\
K_{P}(Q): & =\operatorname{Ker}\left(h^{*}: A(Q) \longrightarrow A(P)\right) .
\end{aligned}
$$

Then $I_{P}(Q)$ and $K_{P}(Q)$ are ideals of $A(Q)$ and

$$
A(Q)=I_{P}(Q) \oplus K_{P}(Q) .
$$

Proof. It follows from Theorem 4 (i) (c), (d) that $K_{P}(Q)$ and $I_{P}(Q)$ are ideals of $A(Q)$. Furthermore since

$$
\begin{array}{ll}
h_{1}: i \longmapsto h(i) & \text { for } \quad i \in P, \\
h^{*}: \delta_{a} \longmapsto \sum_{i \in h^{-1}(a)} \delta_{i} & \text { for } \quad a \in Q,
\end{array}
$$

where $\boldsymbol{\delta}_{a}$ is a primitive idempotent and has the form

$$
\delta_{a}=\sum_{b \leqq a} \mu(b, a) b,
$$


and since $\left\{\boldsymbol{\delta}_{a} \mid a \in Q\right\}$ is a $Z$-basis of $A(Q)$, we have that $K_{P}(Q)$ is generated by elements $\delta_{a}, a \in Q-h(P)$. Clearly, $I_{P}(Q)$ is generated by $h(i), i \in P$. Let $i \in P$. Then by the locally homeomorphism of $h$, we have

$$
\begin{aligned}
\delta_{h(i)} & =\sum_{b \leqq h(i)} \mu(b, a) b \\
& =\sum_{j \leqq i} \mu(h(j), h(i)) h(j) \\
& \in I_{P}(Q) .
\end{aligned}
$$

Thus

$$
\begin{array}{r}
1=\sum_{a \in h(P)} \delta_{a}+\sum_{b \notin h(P)} \delta_{b} \\
\quad \in I_{P}(Q)+K_{P}(Q),
\end{array}
$$

and so $A(Q)=I_{P}(Q)+K_{P}(Q)$. By the Frobenius reciprocity (Theorem 3 (i) (d)), $\left(I_{P}(Q) \cap K_{P}(Q)\right)^{2}=0$. But $A(Q)$ has no non-zero nilpotent elements (Theorem 2), and so $I_{P}(Q) \cap K_{P}(Q)=0$, whence $A(Q)=I_{P}(Q) \oplus K_{P}(Q)$, proving the theorem.

Next we show a transfer theorem which corresponds to the focal subgroup theorem in finite group theory (c. f. Gorenstein [1968, Theorem 7. 3. 4], Cartan-Eilenberg [1956, Theorem 7.10.1], Dress [1974, Proposition 3. 2]). Let $h: P \rightarrow Q$ be a locally homeomorphism. We define a subposet $P_{h}^{n}(n \geqq 0)$ of the direct product $P^{n}$ by

$$
P_{h}^{n}=\left\{\left(x_{0}, \cdots, x_{n-1}\right) \mid x_{i} \in P, h\left(x_{0}\right)=\cdots=h\left(x_{n-1}\right)\right\}
$$

and we denote $p_{i}: P_{h}^{n+1} \rightarrow P_{h}^{n}$ the projection which omits the $i$-th factor, $0 \leqq i \leqq n$. For the convenience, we set $P_{h}^{0}=Q, P_{h}^{1}=P$ and $p_{0}=h: P_{h}^{1} \rightarrow P_{h}^{0}$. Then we have two chain complexes:

$$
\begin{aligned}
& 0 \longrightarrow A(Q) \stackrel{d^{0}}{\longrightarrow} A(P) \stackrel{d^{1}}{\longrightarrow} A\left(P_{h}^{1}\right) \stackrel{d^{2}}{\longrightarrow} \cdots \\
& 0 \longleftarrow A(Q) \longleftarrow d_{0} A(P) \longleftarrow d_{1} A\left(P_{h}^{1}\right) \longleftarrow d_{2} \cdots
\end{aligned}
$$

where $d^{n}=\sum_{i=0}^{n}(-1)^{i} p_{i}^{*}, d_{n}=\sum_{i=0}^{n}(-1)_{i} p_{i !}$.

THEOREM 6. If the locally homeomorphism $h: P \rightarrow Q$ is surjective, then the above sequences are exact.

Proof. We work in the category $\mathscr{O}_{l h} / Q(\cong Q)$. The map $(h: P \rightarrow Q) \mapsto$ $A(P)$ gives a Mackey functor on $\mathscr{O}_{l h} / Q$. Since $h$ is surjective, $h_{1}: A(P) \rightarrow$ $A(Q)$ is also surjective. Thus the theorem follows from Dress theorem 
(Dress [1973, Section 3, Corollary 1], tom Dieck [1979, Proposition 6. 16 and Theorem 6.2.2]).

Span rings. We finally mention a ring which is like to matrix rings, Hecke rings and incidence algebras. Let $G$ be a Green functor on a category $\mathcal{E}$ ([D. 1973]) and $X$ an object of $\mathcal{E}$. Then the abelian group $G(X \times X)$ has another associative ring structure which is called the span ring with respect to $G$ by the following bilinear map :

$$
\begin{aligned}
& G(X \times X) \times G(X \times X) \\
& \begin{array}{r|l} 
& \mid \begin{array}{l}
\pi_{22}^{*} \times \pi_{23}^{*} \\
\times
\end{array}(X \times X \times X \times X \times X)
\end{array} \\
& G(X \times X \times X) \\
& G(X \times X),
\end{aligned}
$$

where $\pi_{i j}$ is the projection to the $(i, j)$-factor. We denote the span ring by $G(X \times X)_{s}$ in order to distinguish it from the ring $G(X \times X)$ with ordinary multiplication of the Green functor. (The ordinary multiplication corresponds to the Hadamard product of matrices).

If $M$ is a Mackey functor on $\mathcal{E}$ and it is furthermore a " $G$-module", then $M(X)$ is a module over the span ring $G(X \times X)_{s}$.

We consider about span rings with respect to Möbius ring functor $A$. Let $h: P \rightarrow Q$ be a locally homeomorphism of finite posets and define a subposet $P \times{ }_{h} P$ of $P \times P$ consisting of pairs $(x, y)$ of elements of $P$ such that $h(x)=h(y)$. Then the multiplication * in the span ring $A\left(P \times{ }_{h} P\right)_{s}$ is given as follows :

$$
(a, b) *(c, d)=\sum_{(x, y) \leqq(a, d)} \sum_{z \leqq b, c} \mu_{Q}(h(x), h(z))(x, y) .
$$

Remember that if $X$ is a $Q$-set corresponding to $h$, then the locally homeomorphism $P \times{ }_{h} P \rightarrow Q$ corresponds to the $Q$-set $X \times X$ and $A\left(P \times{ }_{h} P\right)_{s} \cong$ $G_{0}(Q / X \times X)_{s}$. The action of the span ring $A\left(P \times{ }_{h} P\right)_{s}$ on $A(P)$ is given by

$$
(a, b)^{*} c=\sum_{x \leqq a} \sum_{z \leq b, c} \mu_{Q}(h(x), h(z)) x .
$$

For each $k \in Q$, define a linear map of the span ring to the matrix ring

$$
\psi_{k}: A\left(P \times{ }_{h} P\right)_{s} \longrightarrow \operatorname{Mat}\left(h^{-1}(k) \times h^{-1}(k) ; Z\right)
$$

by 


$$
\phi_{k}(a, b)_{x, y}= \begin{cases}1 & \text { if } x \leqq a \text { and } y \leqq b \\ 0 & \text { otherwise }\end{cases}
$$

Then $\psi_{k}$ is a ring homomorphism and the map

$$
\phi=\left(\phi_{k}\right): A\left(P \times{ }_{h} P\right)_{s} \longrightarrow \prod_{k \in Q} \operatorname{Mat}\left(h^{-1}(k) \times h^{-1}(k) ; Z\right)
$$

is a ring isomorphism.

\section{References}

[A. 1979] M. AIGNER: "Combinatorial Theory", Springer-Verlag, 1979.

[CE. 1956] E. CARTAN-S. Eilenberg: "Homological Algebra," Princeton University Press, 1956.

[B. 1979] K. BACLAWSKI: The Möbius algebra as a Grothendieck ring, J. Algebra, 57 (1979), 167-179.

[Di. 1979] T. tom DIECK: “Transformation Groups and Representation Theory", Springer Lecture Notes in Math., 766, Springer-Verlag, 1979.

[Dr. 1971] A. DRESS: Notes on the Theory of Representation of Finite Groups, Part I, Lecture notes, Bielefeld University, 1971.

[Dr. 1973] A. DRESS: Contributions to the theory of induced representations, in “Algebraic K-Theory II", Proc. Battelle Institute Conference 1972, Springer Lecture Notes in Math., 342 (1973), 183-240.

[Dr. 1974] A. DRESS: On relative Grothendieck rings, in "Representations of Algebras", Proc. International Conference on Representations of Algebras, Ottawa 1974, Springer Lecture Notes in Math, 488, 79-131.

[Go. 1967] D. Gorenstein: "Finite Groups", Harper and Row, New York, 1967.

[Gre. 1973] C. GREENE: On Möbius algebra of a partially ordered set, Advances in Math. 10 (1973), 177-187.

[Gri. 1971] P. A. GRILlet: Regular categories, in "Exact Categories and Categories of Sheaves", Springer Lecture Notes in Math., 236 (1971), 121-222.

[J. 1977] P. T. Johnstone: “Topos Theory”, Academic Press, 1977.

[M. 1971] S. MACLANE: "Categories for the Working Mathematician", SpringerVerlag, 1971.

[S. 1967] L. Solomon: The Burnside algebra of a finite group, J. Combinatorial Theory 2 (1967), 603-615.

[T. 1975] B. R. TEnnison: "Sheaf Theory", London Math. Soc. Lecture Note Series 20, 1975.

[Y. 1983] T. YoshIDA: Idempotents of Burnside rings and Dress induction theorem, J. Algebra 80 (1983), 90-105.

[Y. 198?] T. YosHIDA: When two digraphs are isomorphic ?, to appear.

Department of mathematics

Hokkaido University

Sapporo 060, Japan 